\section{Recipes for success in the laboratory}

\author{
Richard Perham
}

Methods in Molecular Biology, Vols 1

and 2.

Edited by John M. Walker.

Humana/Wiley: 1984/1985. Each volume pp.370, \$45, £41.

A CYNICAL older colleague once advised me that any successful academic would turn out to be one-armed, for only then would he be prevented from saying "On the one hand, this; on the other hand, that". By the same token, the author of a book on methods is confronted with a comparable problem; do I tell my readers about all the different ways to carry out this or that technique or do I limit myself to one which, from personal experience, I know works? Any practising molecular biologist will be aware that the same experimental technique is often carried out differently in different laboratories. The differences may be major, they may be minor: in some instances they will actually matter.

The contributors to these two volumes have, for the most part, opted for the second course. Each method selected for description receives an account replete with experimental detail, taken presumably from the author's laboratory notebooks. There are few caveats, even fewer descriptions of variations or alternatives. There are, of course, further references given for those interested in pursuing them and sometimes a range of useful hints on trouble-shooting. Volume 1 deals with proteins, Vol. 2 with nucleic acids. 1 cannot speak with authority on every method listed - the net has been cast very wide but I could find no serious fault with the recipes for those methods I know well.

I do have some reservations. For example, Vol. 1 has 38 chapters covering techniques from the Lowry method of protein estimation to manual microsequencing, from polyacrylamide gel electrophoresis to hybridoma production. Some room might have been found for the chemical modification of proteins and the methods, enzymic and chemical, of peptide bond cleavage. Even something as basic as the $S$-carboxymethylation of proteins is tucked away in a description of peptide separation by hplc. On the other hand, automated sequencing is wisely eschewed, given the requirement for unusually sophisticated and expensive apparatus.

The second volume is, if anything, more comprehensive, the 53 chapters starting with DNA assay and isolation and ending with DNA sequencing. In between it covers mRNA, cell-free translation, plasmids, cloning in bacteriophage lambda and a host of other things. It was therefore disappointing to find, for example, no description of nucleic acid hybridization

directly in dried agarose gels or of the chemical synthesis of oligonucleotide probes.

But these are quibbles. Molecular biology now permeates so much of present research that a working knowledge of its methods can be essential; it is at once the queen and handmaiden of the biological sciences. The editor tells us that the intention was to collect descriptions of methods that would definitely work, particularly in the hands of novices, and 1 fancy that these books will rightly be welcomed in such quarters. They will not replace the fuller, more discursive treatments given by the originators of these techniques in, say, Methods in

\section{Fields of physics}

\section{Graham G. Ross}

\section{Quantum Field Theory.}

By F. Mandl and G. Shaw.

Wiley: 1984. Pp.354. Hbk £38, \$34.95; pbk $£ 19, \$ 24.95$.

CONSIDERING the importance of the subject, there has been a remarkable shortage of textbooks available for the student of field theory. For many years there were no significant texts to follow the pioneering, and excellent, accounts of Bjorken and Drell, and Schweber, and a very nice short book by Mandl. The situation has improved lately with the encyclopaedic work by Itzykson and Zuber, a very readable text by Ramond covering the path integral formalism and many others. It is in this context that Mandl and Shaw's Quantum Field Theory appears.

A pleasing feature of the book is that it is accessible to the new student, starting immediately with the Lagrangian formalism, and giving a detailed and careful treatment of quantum electrodynamics (QED). I liked the emphasis on performing explicit perturbative calculations and the implementation of the renormalization programme, although I was disappointed that the renormalization group is not discussed. Throughout, the text is augmented with worked examples and problems. It thus provides a welcome update of earlier textbooks on electrodynamics; it is more straightforward than Bjorken and Drell, more detailed than another recent introduction to the subject by Halzen and Martin, and should give both the experimental and theoretical student a good working knowledge of QED.

In its treatment of developments beyond QED the book has some shortcomings. There is no discussion of QCD, the nonAbelian gauge field theory for the strong interactions, and only a relatively short account of the gauge theory for the electroweak interactions. Indeed the concept of gauge invariance, central to modern developments in field theory, is only
Enzymology. Nor perhaps will they have the impact of Cold Spring Harbor's celebrated Molecular Cloning: A Laboratory Manual by Maniatis et al. (now sadly getting out of date) which informed so many beginners in their first fumbling steps in recombinant DNA work some three years ago. However, if, as intended, these books enable those of stout heart but limited experience to gain entry to the growing battery of molecular biological methods, they will have served a useful purpose.

Richard Perham is a Reader in the Department of Biochemistry, University of Cambridge, and President of St John's College, Cambridge.

developed in Chapter 12 and the path integral formalism, which simplifies the analysis of spontaneously broken theories, is not covered. As a result the discussion of the electroweak theory is disappointing, particularly as it does not present a calculation in a spontaneously broken theor: beyond the leading order, nor cover the renormalization in such theories. This renders the book less useful than it might have been for it is in this area that much progress has been made which is not yet adequately covered for the novice graduate student.

Mandl and Shaw have produced a sound introduction to the subject of Abelian gauge field theories which should also be useful as a reference text for perturbative calculations. However it provides only part of a modern introductory course and should be read together with another text, for example that of Ramond, which deals with the advances beyond QED.

Graham G. Ross is in the Department of Theoretical Physics at the University of Oxford.

After writing this review I received a second text on the same subject, Quantum Field Theory by Lewis H. Ryder (Cambridge University Press; $£ 40, \$ 74.50$ ). The similarity to Mandl and Shaw ends with the title, for Ryder provides an introduction to many of the recent advances in field theory, using the path integral formalism to derive Feynman rules for spontaneously broken non-Abelian gauge theories and including a discussion of some of the topological properties and solutions of these theories. The approach differs too, with emphasis on the more formal aspects, no problems and few worked examples. The book is quite readable and is a good introductory text suitable for a theory student, but is probably best read after Ramond's book. In many ways it complements Mandl and Shaw, although the lack of worked examples will be hindrance to the student wanting a full understanding of the subject, and again there is no explicit calculation presented for a spontaneously broken gauge theory. 